\title{
Emergency Department Observation Units: Less Than We Bargained For?
}

\author{
Jahan Fahimi, MD, MPH ${ }^{1 *}$, Renee Y. Hsia, MD, MSc ${ }^{1,2}$
}

${ }^{1}$ Department of Emergency Medicine, University of California, San Francisco, San Francisco, California; 'Institute of Health Policy, University of California, San Francisco, San Francisco, California.

Over the past 3 decades, emergency department observation units (EDOUs) have been increasingly implemented in the United States to supplement emergency department (ED) care in a time of increasing patient volume and hospital crowding. Given the limited availability of hospital resources, EDOUs provide emergency clinicians an extended period of time to evaluate and risk-stratify patients without necessitating difficult-toobtain outpatient follow-up or a short-stay hospitalization. Changes in Medicare and insurer reimbursement policies have incentivized the adoption of EDOUs, and now, over one-third of EDs nationally offer an observation unit. ${ }^{1}$

Much of the observation-science literature has been condition and institution specific, showing benefits with respect to cost, quality of care, safety, and patient satisfaction. ${ }^{2-5}$ Until now, there had not been a national study on the impact of EDOUs to investigate important outcome: hospital admission rates. Capp and colleagues, using the National Hospital Ambulatory Care Survey (NHAMCS), attempt to answer a very important question: Do EDs with observation units have lower hospital admission rates? ${ }^{6}$ To do so, they first standardize admission rates to sociodemographic and clinical features of the patients, while adjusting for hospital-level factors. Then they compare the riskstandardized hospital admission rate between EDs with and without an observation unit as reported in the NHAMCS. The authors make creative and elegant use of this publicly available, national dataset to suggest that EDOUs do not decrease hospital admissions.

The authors appropriately identify some limitations of using such data to answer questions where nuanced, countervailing forces drive the outcome of interest. It is important to note the basic statistical premise that the inability to disprove the null hypothesis is not the same thing as proving that the null hypothesis is true. In other words, although this study was not able to detect a difference between admission rates for hospitals with EDOUs and those without, it cannot be absolutely

*Address for correspondence and reprint requests: Jahan Fahimi, MD, 505 Parnassus Avenue, L126, Box 0209, San Francisco, CA 94143; Telephone: 415-353-1684; Fax: 415-353-3531; E-mail: jahan.fahimi@ ucsf.edu

Received: August 6, 2015; Accepted: August 7, 2015 2015 Society of Hospital Medicine DOI 10.1002/jhm.2475 Published online in Wiley Online Library (Wileyonlinelibrary.com). taken to mean that there is no relationship. The authors clearly state that this study was underpowered given that the difference of ED risk-standardized hospital admission rates was small and therefore is at risk of type II error. In addition, unmeasured confounding may hide a true association between EDOUs and admission rates. Both static and dynamic measures of ED volume, crowding, and boarding, as well as changes in case mix or acuity may drive adoption of EDOUs, ${ }^{7}$ while simultaneously associated with risk of hospitalization. Without balance between the EDs with and without observation units, or longitudinal measures of EDs over time as they are implemented, we are left with potentially biased estimates.

It is also important to highlight that not all EDOUs are created equal. ${ }^{8}$ EDs may admit patients to the observation unit based on prespecified conditions or include all comers at physician discretion. Once placed in observation status, patients may or may not be managed by specific protocols to provide guidance on timing, order, and scope of testing and decision making.

Finally, care in EDOUs may be provided by emergency physicians, hospitalists, or other clinicians such as advanced practice providers (eg, physician assistants, nurse practitioners), a distinction that likely impacts the ultimate patient disposition. In fact, the NHAMCS asks the question, "What type of physicians make decisions for patients in this observation or clinical decision unit?" Capp et al., however, did not include this variable to further stratify the data. Although we do not know whether or not inclusion of this factor may have ultimately changed the results, it could have implications for how distinctions in who manages EDOUs could affect admission rates.

Still, the negative findings of this study seem to raise a number of questions, which should spark a broader discussion on EDOUs. The current analysis provides an important first step toward a national understanding of EDOUs and their role in acute care. Future inquiries should account for variation in observation units and the hospitals in which they are housed as well as inclusion of meaningful outcomes beyond admission rates. A number of methodological approaches can be considered to achieve this; propensity score matching within observational data may provide better balance between facilities with and without EDOUs, whereas multicenter impact analyses using controlled before-and-after or cluster-randomized trials should be considered the 
gold standard for studying observation unit implementation. Outcomes in these studies should include long-term changes in health, aggregate healthcare utilization, overuse of resources that do not provide high-value care, and impacts on how care and costs may be redistributed when patients receive more care in observation units.

Although cost containment is often touted as a cornerstone of EDOUs, it is critical to know how the costs are measured and who is paying. For example, when an option to place a patient in observation exists, might clinicians utilize it for some patients who do not require further evaluation and testing and could have been safely discharged?" This "observation creep" may arise because clinicians can use EDOUs, not because they should. Motivations may include delaying difficult disposition decisions, avoiding uncertainty or liability when discharging patients, limited access to outpatient follow-up, or a desire to utilize observation status to justify the existence of EDOUs within the institution. In this way, EDOUs may, in fact, provide low-value care at a time of soaring healthcare costs.

Perhaps even more perplexing is the question of how costs are shifted through use of EDOUs. ${ }^{10,11}$ Much of the literature advertising its cost savings are only from the perspective of the insurers' or hospitals' perspective, ${ }^{12}$ with 1 study estimating a potential annual cost savings of $\$ 4.6$ million for each hospital, or $\$ 3$ billion nationally, associated with the implementation of observation care. ${ }^{5}$ But are medical centers just passing costs on to patients to avoid penalties and disincentives associated with short-stay hospitalizations? Both private insurers and the Centers for Medicare and Medicaid Services may deny payments for admissions deemed unnecessary. Further, under the Affordable Care Act, avoiding hospitalizations may mean fewer penalties when Medicare patients later require admission for certain conditions. As such, hospitals may find huge incentives and cost savings associated with observation units. However, using EDOUs to avoid the Medicare readmission penalty may backfire when less-sick patients requiring care beyond the ED are treated and discharged from observation, leaving more medically complex and ill patients for hospitalization, a group potentially more likely to be rehospitalized within 30 days, making readmission rates appear higher.

Nonetheless, because services provided during observation status are billed as an outpatient visit, patients may be liable for a proportion of the overall visit. In contrast to inpatient stays where, in general, patients owe a single copay for most or all of services rendered, outpatient visits typically involve a la carte billing. When accounting for costs related to professional and facilities fees, medications, laboratory tests, and advanced diagnostics and procedures, patient bills may be markedly higher when they are placed in observation status. This is especially true for patients covered by Medicare, where observation stays are not covered under Part A.

Research will need to simultaneously identify best practices for how EDOUs are implemented and administered while appraising their impact on patient-centered outcomes and true costs, from multiple perspectives, including the patient, hospital, and healthcare system. There is reason to be optimistic about EDOUs as potentially high-value components of the acute care delivery system. However, the widespread implementation of observation units with the assumption that it is cost saving to hospitals and insurers, without high-quality population studies to inform their impact more broadly, may undermine acceptance by patients and health-policy experts.

Disclosure: Nothing to report.

\section{References}

1. Wiler JL, Ross MA, Ginde AA. National study of emergency department observation services. Acad Emerg Med. 2011;18(9):959-965.

2. Baugh CW, Venkatesh AK, Bohan JS. Emergency department observation units: a clinical and financial benefit for hospitals. Health Care Manag Rev. 2011;36(1):28-37.

3. Goodacre S, Nicholl J, Dixon S, et al. Randomised controlled trial and economic evaluation of a chest pain observation unit compared with routine care. BMJ. 2004;328(7434):254.

4. Rydman RJ, Roberts RR, Albrecht GL, Zalenski RJ, McDermott M. Patient satisfaction with an emergency department asthma observation unit. Acad Emerg Med. 1999;6(3):178-183.

5. Baugh CW, Venkatesh AK, Hilton JA, Samuel PA, Schuur JD, Bohan JS. Making greater use of dedicated hospital observation units for many short-stay patients could save $\$ 3.1$ billion a year. Health Aff (Millwood). 2012;31(10):2314-2323.

6. Capp R, Sun B, Boatright D, Gross C. The Impact of emergency department observation units on U.S. emergency department admission rates. J Hosp Med. 2015;10(11):738-742.

7. Hoot NR, Aronsky D. Systematic review of emergency department crowding: causes, effects, and solutions. Ann Emerg Med. 2008;52(2): $126-136$.

8. Mace SE, Graff L, Mikhail M, Ross M. A national survey of observation units in the United States. Am J Emerg Med. 2003;21(7): 529-533.

9. Crenshaw LA, Lindsell CJ, Storrow AB, Lyons MS. An evaluation of emergency physician selection of observation unit patients. Am J Emerg Med. 2006;24(3):271-279.

10. Ross EA, Bellamy FB. Reducing patient financial liability for hospitalizations: the physician role. J Hosp Med. 2010;5(3):160-162.

11. Feng Z, Wright B, Mor V. Sharp rise in Medicare enrollees being held in hospitals for observation raises concerns about causes and consequences. Health Aff (Millwood). 2012;31(6):1251-1259.

12. Abbass IM, Krause TM, Virani SS, Swint JM, Chan W, Franzini L. Revisiting the economic efficiencies of observation units. Manag Care. 2015;24(3):46-52. 